\title{
WAVE DYNAMICS AND STAR FORMATION IN TAURUS
}

\author{
Ralph E. Pudritz and Ana I. Gomez de Castro \\ Dept. of Physics, McMaster University, Hamilton, ON L8S 4M1
}

\section{Introduction}

The mechanism underlying the formation of cores and larger scale structures in molecular clouds must play a fundamental role in the physics of star formation since young stellar objects are usually found within or very near cores (Myers et al 1987, Beichman et al 1986). The Taurus cloud is an ideal object to study in this regard because of its proximity ( $160 \mathrm{pc})$, and because only low mass star formation is presently occurring there. Barnard's (1927) beautiful optical photograph of the region reveals that the obscuring gas and dust has filamentary structure that is comparable to the size of the cloud complex (several 10's of pc). This structure is clearly seen in CO maps of the region as well (eg. Duvert et al 1986) where it is apparent that structure on much larger size scales than cores is common. In addition to the filamentary structure one also observes that there are small dark clouds present such as L1489, L1495, etc.

The CO clumps in the Taurus cloud and others appear to be like raisins embedded within the stringy filaments. The dense $\mathrm{NH}_{3}$ cores mapped by Benson and Myers (1989) however, are concentrated in the dark clouds (such as L1495). It is standard procedure to characterize the mass distribution of clumps and cores in molecular clouds by fitting the number of clumps per unit mass range with a power law spectrum; $d N(m) / d m \propto m^{-\alpha}$. We have done this for the collection of the 14 $\mathrm{NH}_{3}$ cores in Taurus that were studied by Benson and Myers (1989) and find that the core mass spectrum has the value $\alpha \simeq 1.5$. This is somewhat different value than has been determined for $\rho$ Oph where $\alpha \simeq 1.1$ (Loren 1989) but similar to that for the Rosette nebula $\alpha \simeq 1.5$ (Blitz 1989). It is presently unclear whether or not these differences in core mass spectra from cloud to cloud reflect experimental uncertainty or real differences in the physics or evolutionary states of the different well studied clouds. We present simulations which indicate that the structure and mass spectra of clouds is expected to evolve rather strongly during the life of a molecular cloud.

In accounting for the large scale features of the Taurus clouds we can rule out the damaging effects of low mass star formation within the cloud (such as the production of cavities and shells blown by the bipolar outflows) since such regions would not exceed a few pc. is size. It is therefore likely that the large scale structure in Taurus was formed by global processes involving the whole cloud. An important point in this regard is that the Taurus region appears to be associated with a bubble in the HI survey of Heiles and collaborators (eg. Kulkarni and Heiles, 
1988). Expanding shells are likely to break up into filaments and this may be a factor in understanding the Taurus cloud. There are several other important clues about the origin of global structure in Taurus. The HI study of Shuter et al (1987) shows that the velocity field of the region as determined by $21 \mathrm{~cm}$ observations (in absorption) has a wavelike character with a wavelength of order $10 \mathrm{pc}$ and a mean velocity of $2.7 \mathrm{~km} \mathrm{~s}^{-1}$. The magnetic field in Taurus as revealed by optical polarimetry (Moneti et al 1984, Heyer et al 1987) is also ordered on the scales of 10 's of pc. In fact there is a distinct "waviness" to the field. Since the magnetic field is comparable to gravitational energy densities (see eg Heiles, these proceedings, Myers and Goodman 1988) the observational evidence supports the idea that hydromagnetic waves whose wavelength is comparable to cloud size, may in fact be playing an important role in global cloud physics.

What would be explained if such waves (either nonlinear Alfvén waves or even linear fast magnetosonic waves) were present? There are at least four important aspects of cloud dynamics and structure of interest:

(i) long wavelength transverse waves can in principle stir up the largest scales in the cloud and thereby provide support against rapid global collapse (Arons and Max 1975),

(ii) scales intermediate between the Jeans length in the cloud and the cloud scale can still collapse for plausible wave spectra (Bonazzola et al 1987, Pudritz 1990),

(iii) non-linear Alfvén waves and even linear fast magneto-sonic modes can produce density fluctuations, which in principle could be identified with structure observed on supra Jeans scales (Carlberg and Pudritz 1990),

(iv) large scale waves with periods of several million years would constantly change the apparent alignment of the cloud magnetic field direction with respect to the major axis of the filaments thereby accounting for the fact that these alignments can be found to vary from filament to filament in Taurus, and indeed other molecular clouds.

The last point on this list is rather important in that the lack of alignment between field and filament directions has been interpreted by some authors to imply that the magnetic field on these scales is not dynamically important for cloud physics (eg Heyer 1988), the implication being that ambipolar diffusion has weakened the field substantially by time densities of $10^{4} \mathrm{~cm}^{-3}$ have been achieved. However, since waves on the scales of cores must damp while on larger scales they may propagate for nearly the life of the cloud, one expects misalignments in clouds stirred by hydromagnetic waves!

In this contribution, we demonstrate using linear instability analysis that wave spectra strongly affect the stability of molecular clouds. We then present recent numerical simulations on the effect that a single long wavelength mode has upon the formation of large scale structure in a cloud that is supported against collapse by a subcritical, isotropic magnetic field.

\section{Stability of Clouds Traversed by Hydromagnetic Waves}

The physics of wave propagation in molecular clouds has been recently extensively discussed (eg. Pudritz 1990, Carlberg and Pudritz 1990, henceforth CP) and only the main points will be reviewed here. An essential point is that not all physical scales in a molecular cloud can be supported by the pressure of hydromagnetic waves. Waves of short enough wavelength damp by ion-neutral friction. Magnetic force is only communicated to the neutrals by the intermediary of ion-neutral col- 
lisions. The neutrals will suffer the effect of magnetic forces only if collisions with ions are sufficiently frequent. If we imagine ions to be "riding" an Alfvén wave, then if a given neutral does not collide with any ion in a wave period, large velocity differences between the ions and the neutrals are established so that friction between the ion and neutral "fluids" are large (the latter depends upon the relative velocity between the two components). Large friction always means wave damping and so one finds the critical condition (Braginskii 1965, Kulsrud and Pearce 1969) that Alfvén waves with angular velocities larger than $2 \omega_{n, i}$ damp completely where $\omega_{n, i}=0.50 \times 10^{-14}\left(n_{3} \zeta_{-17}\right)^{1 / 2} \mathrm{~s}^{-1}$ is the frequency with which a given neutral collides with any ion. The associated damping length $\lambda_{\min }$ is such that all waves with $\lambda<\lambda_{\min }$ completely damp, while larger wavelength waves can propagate suffering some damping as they go. The ratio of this damping length to the non-magnetic Jeans length in the gas is

$$
\frac{\lambda_{\min }}{\lambda_{J}}=0.62 \frac{V_{o}}{5 c_{s}} \frac{B}{B_{c}} \zeta_{-17}^{-1 / 2}
$$

where the virial speed $V_{o}$ in a molecular cloud is typically five times the sound speed, $B_{c}$ is the critical magnetic field strength in a molecular cloud (about $30 \mu \mathrm{G}$ ) that could support the cloud against gravitational collapse, and where $\zeta_{-17}$ is the ionization rate per hydrogen atom measured in units of $10^{-17} \mathrm{~s}^{-1}$ per hydrogen atom. Thus for clouds that are magnetically subcritical, the damping length for waves is somewhat larger that the Jeans length and of order the observed size of individual cores seen in molecular clouds. For supercritical clouds, the damping scale is less than the Jeans length so effectively all supra Jeans scales could be stirred ( but not supported ) by hydromagnetic waves. The damping scale is roughly the size of cores observed in molecular clouds, and CP proposed that a likely reason why cores form is that wave pressure is unavailable to support gas on scales $\lambda_{J}<$ $\lambda<\lambda_{\min }$. Thus magnetically subcritical clouds start to produce cores on a scale determined by the magnetic damping length.

The time scale for the damping of linear waves is

$$
\tau_{D}=3.2 \times 10^{6} \frac{1}{\left(n_{3} \zeta_{-17}\right)^{1 / 2}}\left(\frac{\lambda}{\lambda_{\min }}\right)^{2} y r
$$

which is very sensitive to the wavelength. Applying equation (2.) to the scales characterizing cores $\left.\left(n_{3}=10, \lambda \simeq \lambda_{\min }\right)\right)$, we find that wavelengths of order the core size damp in $1.0 \times 10^{6} \mathrm{yr}$ whereas long wavelength waves of order the size of the cloud $\lambda \simeq 10 \lambda_{\min }$ damp in $3.2 \times 10^{8} \mathrm{yr}$. Thus, while the longest modes in a cloud "ring" for the life of the cloud, modes on scales of cores damp in about a cloud free-fall time (a million years). The energy losses on small scales do not pose a great problem for maitaining waves in the cloud since the power dissipated on the scales of cores represents only $1 \%$ of the power in the wave field at the longest wavelengths (most of the power must be found at the largest scales if global gravitational collapse is to be staved off). The wave power dissipated on the scale of cores in fact heats such regions to temperatures of $10-20 \mathrm{~K}(\mathrm{CP})$. Thus, both the formation of cores, and their heating are natural consequences of wave damping in molecular clouds.

We now turn to consider the question of the stability of molecular clouds which are traversed by a spectrum of MHD waves (see Pudritz 1990). Bonazzola 
et al (1987) were the first to point out that a spectrum of waves with enough power on the largest scales could stir up the gas sufficiently to prevent global gravitational collapse of the cloud. While these authors based their argument on the virial theorem, one can derive the results in a straightforward fashion from the equations of motion of a cloud that is traversed by slowly varying (spatially and temporally) waves. In such a situation, it is well known that the background wave field contributes an additional stress on the fluid, $-\nabla . \mathbf{P}_{\mathbf{w}}$ where $\mathbf{P}_{\mathbf{w}}$ is the wave pressure tensor. Introducing this into the equations of motion describing a molecular cloud, and linearizing, one derives the dispersion relation for waves in warm, partially ionized, self gravitating, magnetized gas stirred by a background wave field,

$$
\omega_{ \pm}=\frac{i}{2}\left(-\frac{\omega_{A, n}^{2}}{\omega_{n, i}} \pm\left[\left(\omega_{A, n}^{4} / \omega_{n, i}^{2}\right)+4 \Omega_{n}^{2}\right]^{1 / 2}\right)
$$

where $\omega_{A, n}$ is the angular velocity of Alfvén waves in the neutral gas. In this expression, the Jeans function $\Omega_{n}^{2} \equiv \omega_{g}^{2}-k^{2}\left[V_{A}^{2}+c_{s}^{2}+\delta v_{A}(k)^{2}\right]$ shows that gravitational instability (the gravitational response frequency is $\omega_{g} \equiv\left(4 \pi G \rho_{n}\right)^{1 / 2}$ ) is offset by the mean magnetic field (whose associated Alfvén speed is $\left.V_{A}\right)$, gas pressure $\left(c_{s}\right.$ is the sound speed in the cloud), and wave pressure (the amplitude of the Alfvén velocity fluctuations due to a spectrum of waves is assumed to obey a power law, $\left.\delta v_{A}(k)=V_{A}\left(k_{o} / k\right)^{\beta}\right)$. The dispersion relation (3.) reduces, in the absence of gravity, gas pressure, and background MHD waves, to that first discovered by Kulsrud and Pearce (1969) for the propagation of Alfvén waves in partially ionized gas. Gravitational instability sets in for any scale for which $\Omega_{n}^{2}>0$ exactly as the Jeans analysis would suggest. One difference with the traditional Jeans result is that the growth time for the unstable modes is strongly modified by the rate of ambipolar diffusion of the magnetic field out of the gas.

The major consequence of having a spectrum of waves stabilize the cloud is that the bandwidth of stable scales depends upon the index $\beta$ of the wave spectrum. One readily finds from (3.) that for wave spectra with $\beta>1$, the largest scales in the cloud are stabilitzed against collapse. Figure 1 is a plot of the growth time scale of an unstable mode as a function of the mode wavelength in the case that the energy density in the waves equals that of gravity $\left(a \equiv \omega_{g}^{2} / k_{o}^{2} V_{A}^{2}=1\right)$. The scale $\lambda_{o}$ is taken to be the size of the molecular cloud and the instability time scale is in units of $\omega_{g}^{-1}=0.72 \times 10^{6} n_{3}^{-1 / 2} \mathrm{yr}$. As one increases the spectral index $\beta$, there is a steeper fall off in the wave energy as one goes to smaller scales. This in turn implies that there is an increasingly larger band width of scale lengths that are without support by wave pressure, and hence gravitationally unstable.

\section{Nonlinear Effects: Simulations of Single Alfvén Modes}

The effect of a spectrum of large amplitude Alfvén modes on a molecular gas is strikingly reminiscent of structure observed in Taurus. Simulation of CP showed that small scale fragmentation of the cloud was later followed by the appearance of larger scale, filamentary structure. The mass spectrum of clumps in the CP simulations constantly evolved to smaller values of $\alpha$, the reason being that significant agglomeration of smaller lumps into larger entities occurs. The clumps that appeared reasonably obeyed Larson's relations.

In order to more fully understand the effect that finite amplitude Alfvén waves can have on molecular clouds, we have completed a number of numerical 


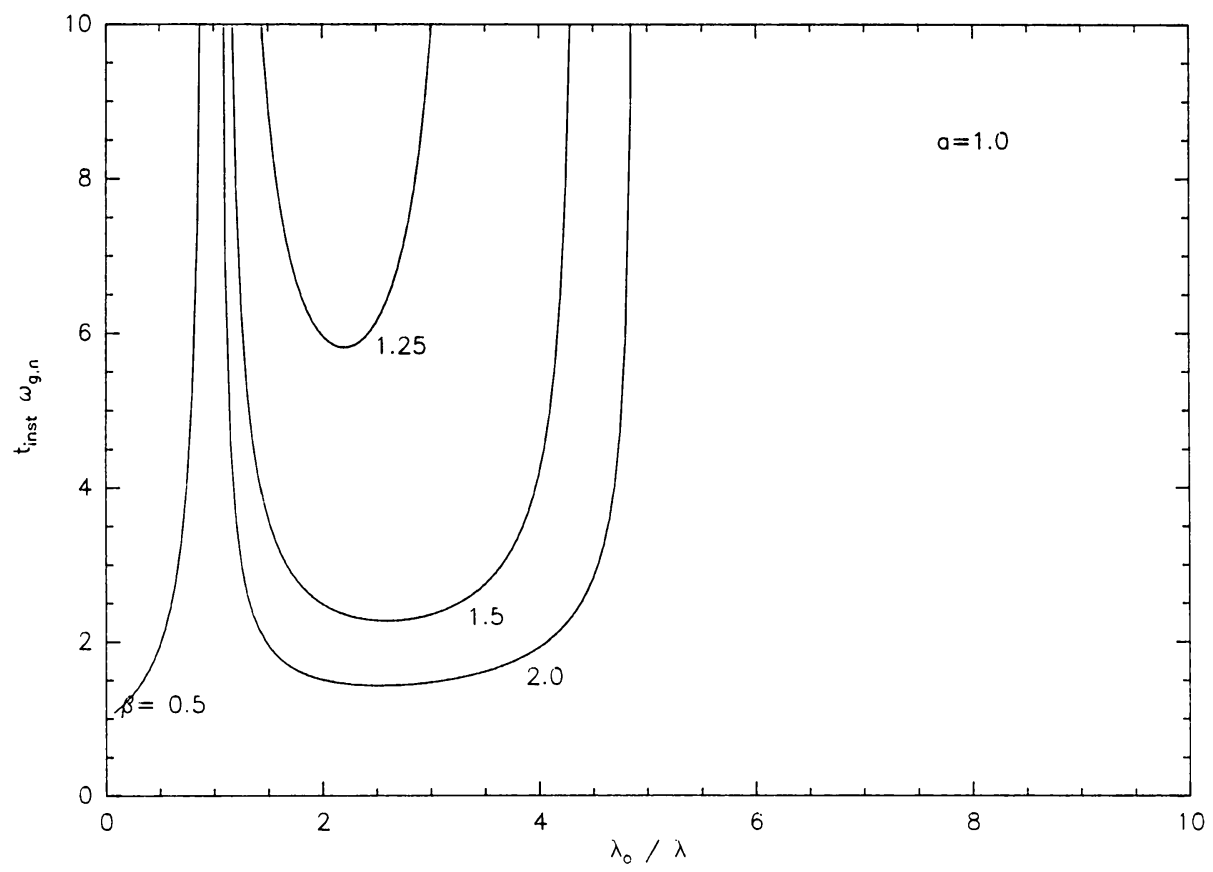

Figure 1. Growth times of modes in clouds stirred by power law wave spectra; the case of $a=1$ (see text); adapted from Pudritz (1990).

experiments in which the evolution of a cold molecular cloud supported by strong isotropic field is compared with an identical model which has a single, long wavelength, Alfvén wave propagating along the z-direction in the model as an additional ingredient. We employed the 3-D, N-body, gas dynamics code ("sticky particles") described in CP and Pudritz and Carlberg (1989) to which we refer the reader for the details.

In Figure 2, we show a comparison of two simulations, one without any waves and one with a single Alfvén mode whose energy was only $25 \%$ of the gravitational energy density. The Jeans length in this cold cloud calculation is $\lambda_{J} / R=0.081$ while the wavelength of the single mode we studied is $\lambda / R=0.33$, where $R$ is the initial radius of the cloud. In all calculations, the initial state of the cloud is a uniform sphere. Slow contraction of the cloud occurs because of ambipolar diffusion. Our models evolve quasi-statically because our initial state is strongly subcritical. The Figure shows quite plainly that large scale structure is much more apparent in the single long mode calculation than in the mode-free model. Evidently, the large scale wave has been resposible for gathering together many small fragments into larger, filamentary entities.

This impression is made precise in Figure 3 which compares the clump mass spectra in the no-mode versus long-mode simulations. The figure shows histograms 

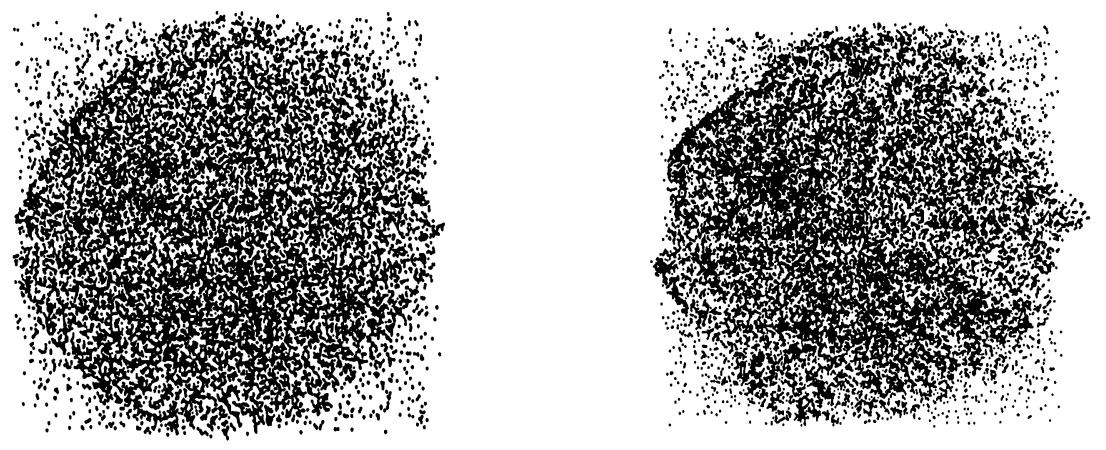

Figure 2. Comparison of simulations of magnetically supported, cold molecular clouds with (a.) no Alfvén wave and (b.) with a single long wavelength mode. These figures show the two respective simulations after $\simeq 4$ free-fall times. The nonmagnetic Jeans length in the cloud is tiny ( 0.081 of the cloud's original radius).

of the clump masses at different times (a cloud free-fall time is 0.4 time units). In both cases, the cloud breaks up into fragments whose mass is nearly the Jeans mass, corresponding to the strong peak at mass $m=3.3 \times 10^{-4} M_{c}$. As time goes by, we see that power appears at the larger mass scales at the expense of power on the smallest scale; ie, agglomeration is occurring. However, agglomeration is not the whole picture. By the end of the calculation, the long mode calculation has significantly more power on the largest scales than does the no-mode simulation. Clearly the long wavelength wave has swept together gas and created significantly larger scale structure than a model in which long wavelengths are absent. This illustrates the basic point namely, that waves generate structure.

In order to give better insight into how larger scale structure develops, we show in Figure 4 the spatial distribution of clumps with a minimum overdensity of 30 over the mean cloud density at two different times, namely at 3 and 3.5 cloud free-fall times. We project these clump positions onto the three different planes $x-z$, $\mathrm{x}-\mathrm{y}$, and $\mathrm{y}-\mathrm{z}$. One sees the very striking amount of agglomeration that is occurring between these two time steps. It is apparent that while a filamentary pattern is difficult to discern at $t=1.2=3 t_{f f}$ it is clearly emerging a half free-fall time later. The reason for the delay in the formation of the larger scale structure is also clear; the wave period of an Alfvén wave is directly proportional to its wavelength, so larger scale filamentation should start in at about 4 free-fall times for our particular choice of a mode.

Finally, we present a log-log plot of the number of clumps $N(m)$ vs the mass of the clump as shown in Figure 5. This again illustrates the trend of spectral evolution of the mass spectrum of clumps, with a new feature. One notes that 

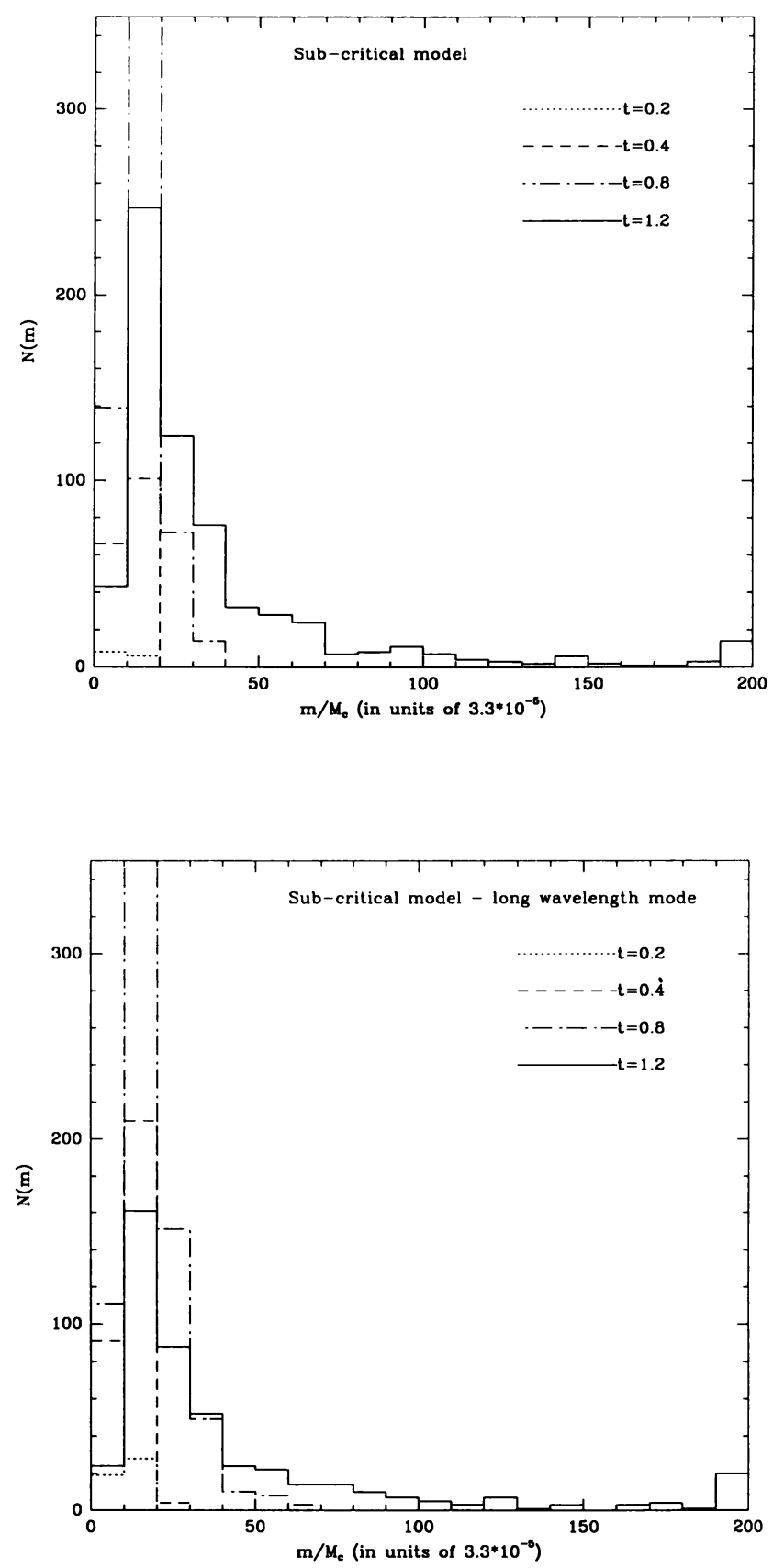

Figure 3. Mass spectra of no mode versus single long wavelength modes in magnetically subcritical cloud. One free fall time is 0.4 time units. 


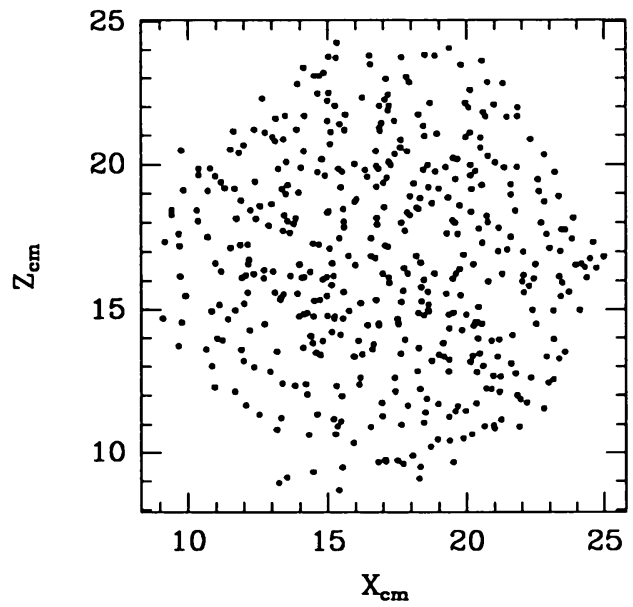

Sub-critical model

long wavelength mode

$t=1.2$
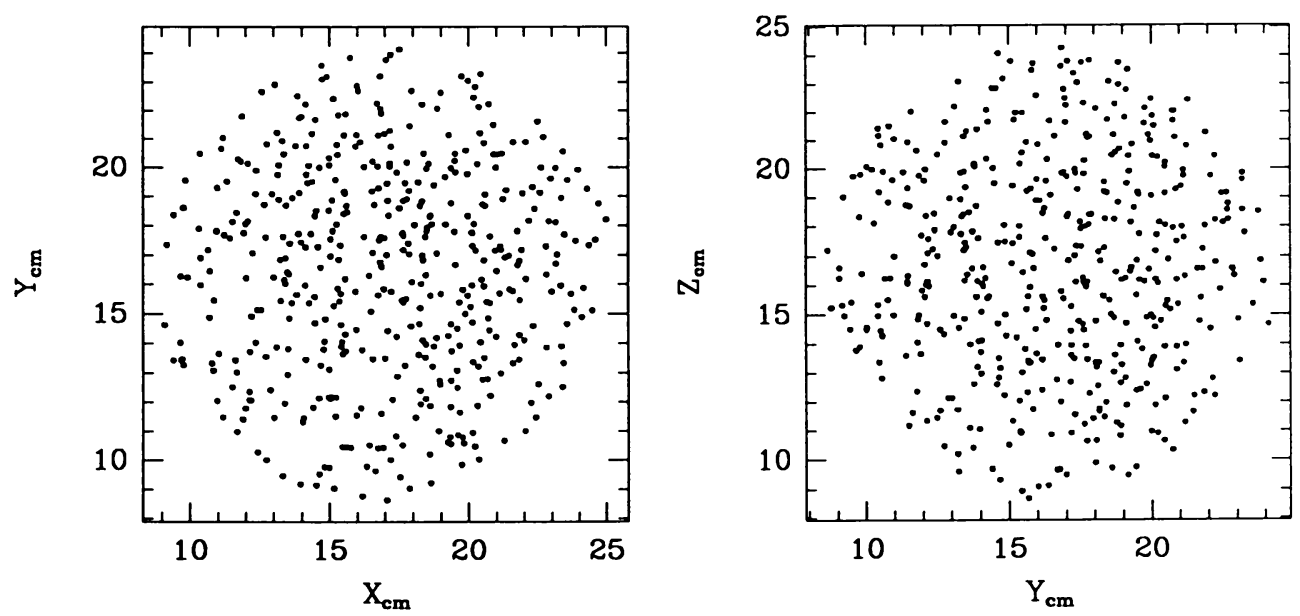

Figure 4a. Evolution of structure in long wavelength mode simulation; projected spatial distribution of clumps with minimum overdensity of 30 at 3 free-fall times.

the spectrum appears to turn over at the small mass end. While something like this has been seen observationally, we note that it is an expected feature of clouds which first fragment into small pieces. We also see that the slope of the log-log plot becomes ever shallower. We have not yet found a model which reaches steady state. This suggests that the spectral index constantly evolves in real clouds, so that cloud to cloud differences may be a real effect, possibly reflecting cloud age.

\section{Origin and Evolution of Cloud Structure}

Given the evidence that there may be long wave-length waves sloshing around inside the Taurus cloud, what may have been the likely origin for such phenomena? Of course there are many possibilities; any agent that shakes a molecular cloud will 


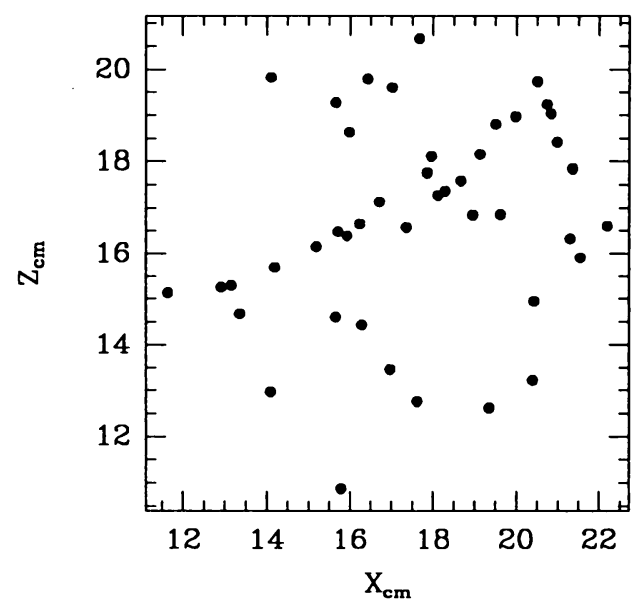

Sub-critical model long wavelength mode $t=1.4$
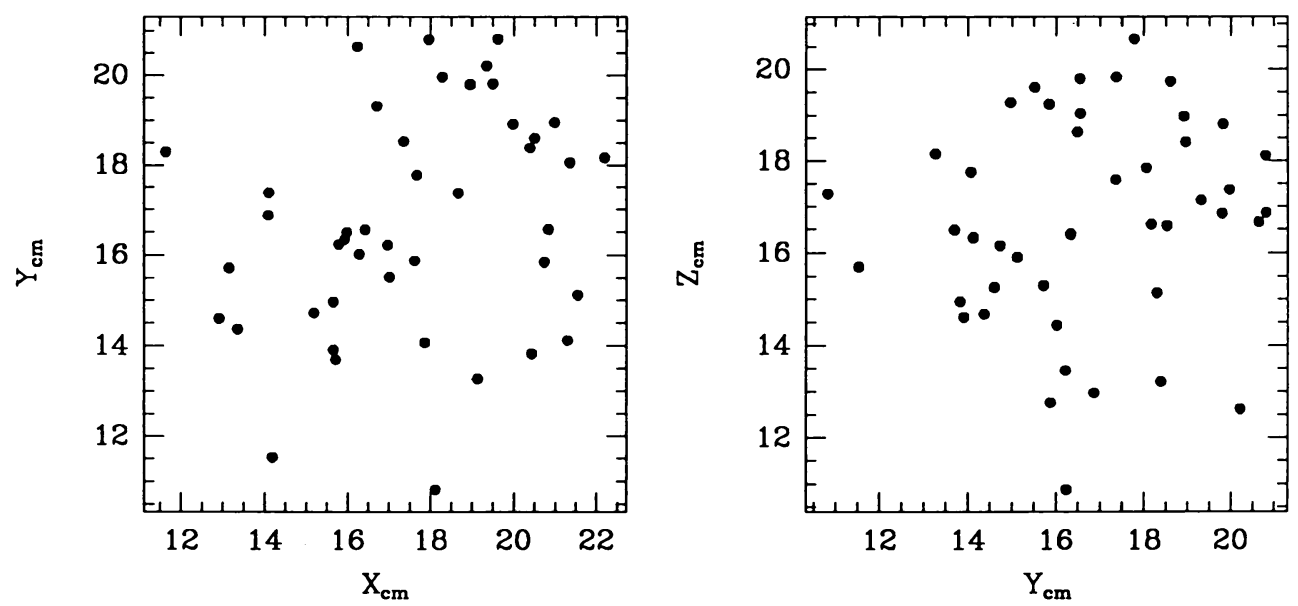

Figure 4b. Same as Figure $4 \mathrm{a}$ except at 3.5 free-fall times.

set off a train of Alfvén waves. However, given the long-livedness of the largest wavelengths, it is entirely possible that large scale wave power has something to do with the formation mechanism of a molecular cloud. We propose that in fact such modes are produced during the process of cloud formation by the Parker-Jeans instability (see eg Blitz and Shu 1979, and Elmegreen 1982). The nonlinear development of the Parker instability consists of gas flowing down the field lines back to the galactic plane, and there colliding with the gas in the magnetic valley. This must lead to oscillations and shocks, as recent numerical experiments have confirmed (eg. Matsumoto et al 1989). Thus the very act of forming a molecular cloud is likely to produce sufficient large scale wave power to keep it supported against subsequent gravitational collapse (Gomez de Castro and Pudritz, in preparation).

Thus, we arrive at the view that it is highly likely that molecular cloud structure evolves rather strongly over the life of a cloud, and that hydromagnetic waves 


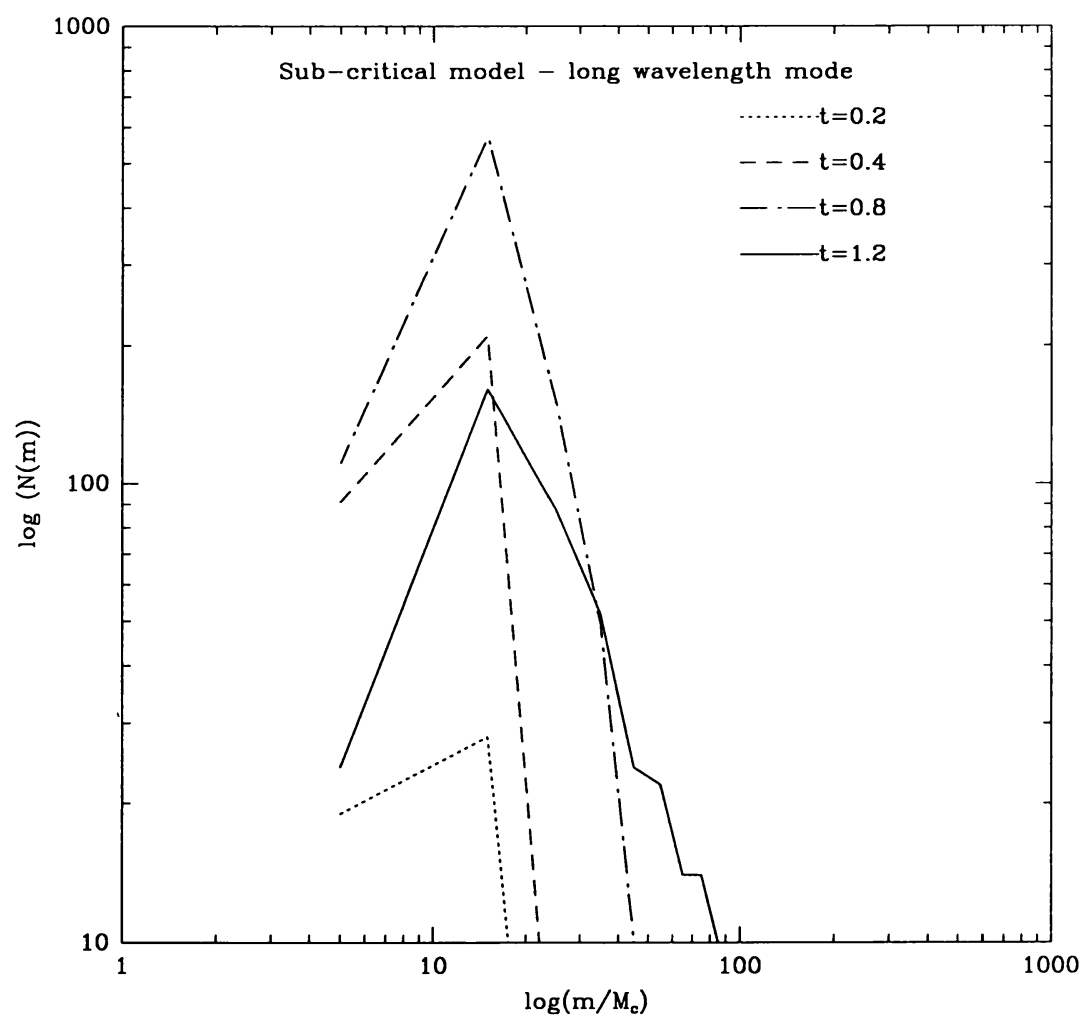

Figure 5. Log-log plot of clump mass spectrum of long-mode simulation.

play an essential role in cloud physics. A sequence of events that summarizes this process is presented below:

1. A Jeans-Parker instability triggers the formation of a molecular cloud or cloud complex; long wavelength modes generated in this process stir the newly forming clouds for their subsequent life.

2. Since molecular clouds are very cold, the smallest fragments are much below the mass of the cloud; the preliminary cloud fragmentation produces pieces of order the Jeans length $\lambda_{J}$ or alternatively $\lambda_{\text {min }}$.

3 . The larger scale modes sweep together larger scale structures several freefall times into the life of the cloud. Agglomeration of the small pieces occurs all the while.

4. The filaments are themselves temporary structures; they end up by draining into the most massive clumps, which ultimately dominate the gravitational field of the cloud.

5. Since the agglomeration time of the clumps is as short as the free-fall time, star formation in the clumps is always disrupted by subsequent merger with other clumps. This implies that star formation does not predominantly occur in small cores scattered through the volume of the cloud, but rather within the most massive clumps that are formed the agglomeration process. Thus a cluster mode of star formation is favoured (see E. Lada's and P. Myers contributions, these 
proceedings).

6. The Taurus cloud may be quite young; it may yet form a massive clump(s) in which a cluster will form. A good candidate for the site of a future cluster is within L1495.

7. The spectral index $\alpha$ characterizing the mass spectrum of clumps in a cloud likely evolves over the life of a cloud; its value may correlate with the age of a cloud.

We are indebted to Ray Carlberg and Patricia Monger for many stimulating conversations and help in implementing some changes of our numerical code. It is a pleasure to thank Edith Falgarone and the scientific organizing committee for their invitation to speak at this interesting meeting, and for financial support given towards this end. This research was supported by NSERC of Canada.

\section{REFERENCES}

Arons, J., and Max, C.E. 1975, Ap. J., 196, L77.

Barnard, E.E. 1927, in Carnegie Inst. of Washington Publications No. 247,I

Beichman, C.A., Myers, P.C., Emerson, J.P., Harris, S., Mathieu, R., Benson, P.J., and Jennings, R.E. 1986, Ap. J., 307, 337.

Benson, P.J., and Myers, P.C. 1989, Ap. J. Suppl., 71, 89.

Blitz, L. 1987, in Physical Processes in Interstellar Clouds, G.E. Morfill and M. Scholer eds. (Kluwer: Dordrecht) 35

Blitz, L. and Shu, F. 1980, Ap. J., 238, 148.

Bonazzola, S., Falgarone, E., Heyvaerts, J., Perault, M., and Puget, J.L. 1987, Astr. Ap., $172,293$.

Braginskii, S.I. 1965, in Reviews of Plasma Physics, Vol 1, M.A. Leontovich ed. (New York: Consultants Bureau)205

Carlberg, R.G., and Pudritz, R.E. 1990, M. N. R. A. S., , in press.

Duvert, G., Cernicharo, J., and Baudry, A. 1986, Astr. Ap., 164, 349.

Elmegreen, B.G. 1982, Astr. Ap., 253, 655.

Heyer, M.H. 1988, Ap. J., 324, 311.

Heyer, M.H., Vrba, F.J., Snell, R.L., Schloerb, F.P., Strom, S.E., Goldsmith, P.F., and Strom, K.M. 1987, Ap. J., 321, 855.

Kulkarni, S.R., and Heiles, C. 1988, in Galactic and Extragalactic Radio Astronomy, G.L. Verschuur and K.I. Kellermann eds. (Springer-Verlag: New York)95

Kulsrud, R., and Pearce, W.P. 1969, Ap. J., 156, 445.

Loren, R.B. 1989, Ap. J, 338, 902, 925.

Matsumoto, R, Horiuchi, T., Hanawa, T., and Shibata, K. 1989, Pub. Astr. Soc. Japan,

Moneti, A., Pipher, J.L., Helfer, H.L., McMillan, R.S., and Perry, M.L. 1985, Ap. J., 282, 508.

Myers, P.C., and Goodman, A. 1988, Ap. J., 326, L27.

Myers, P.C., Fuller, G.A., Mathieu, R.D., Beichman, C.A., Benson, P.J., Schild, R.E., and Emerson, J.P. 1987, Ap. J., 319, 340.

Pudritz, R.E. 1990, Ap. J., 350, 195.

Shuter, W.L., Dickman, R.L., and Klatt, C. 1987, Ap. J., 322, L103. 